\title{
De lectores a creadores de discurso político. Participación ciudadana en Twitter en las campañas electorales del PSC-PSOE (2010-2012)
}

institucional.us.es/ambitos/

\section{Daniel Franco Buendía}

Universitat de Vic

dfranb@gmail.com

\section{Resumen}

Esta investigación estudia la relación existente entre PSC-PSOE, los ciberactivistas afines al partido y la sociedad civil a partir de los mensajes publicados en Twitter por el partido y sus líderes, en las campañas electorales transcurridas entre 2010

y 2012 en Cataluña. Para realizarla, se utiliza una triangulación metodológica compuesta por un análisis de contenidos cuantitativo y cualitativo de los mensajes publicados en Twitter en la cuenta del partido y en los perfiles de los candidatos a las elecciones estudiadas, una revisión bibliográfica y entrevistas en profundidad a los responsables de comunicación 2.0 de PSC-PSOE en las elecciones del periodo analizado. También cuenta con entrevistas semiestructuradas a expertos en

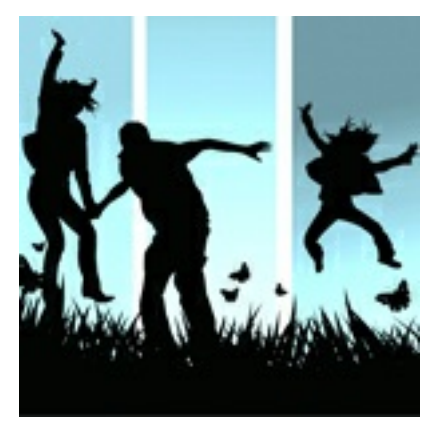

comunicación política 2.0 .

\section{Palabras clave}

Twitter, participación, ciudadanía, PSC-PSOE, elecciones.

Abstract

This research studies the relationship between PSC-PSOE, the cyber activists related to the political party and the civil society through the messages posted on Twitter by the party and its leaders, in the election campaigns held between 2010 and 2012 in Catalonia. For this procedure, we use a methodological triangulation consisting

of ananalysis of quantitative and qualitative content of the messages posted on Twitter in the account of the political party and in the studied profiles of the election candidates, a bibliographic review and in-depth interviews to those responsible for the 2.0 communication of PSC-PSOE in the elections of the analyzed period. There are also semi-structured interviews to experts in political communication 2.0.

\section{Keywords}

Twitter, participation, citizenship, PSC-PSOE, elections.

\section{INTRODUCCIÓN}

En tan solo dos años, los catalanes pudieron manifestar hasta en 4 ocasionessu opinión en las urnas. De 2010 a 2012 se celebraron dos elecciones al Parlament de Catalunya, se eligió a los representantes municipales y a los miembros del Congreso de los Diputados. Todo ello acontece al mismo tiempo que el President de la Generalirat de Catalunya es denunciado, hecho al que se añade la protesta del 15M, una Huelga General y la celebración multitudinaria del día 11 de septiembre de 2012 (1).

Esta voluntad de participación de la ciudadanía y de manifestar su opinión, la recoge el PSC-PSOE en el proceso de regeneración que está sufriendo actualmente. El 6 de abril de 2014, Jaume Collboni sale elegido como candidato del PSC-PSOE al Ayuntamiento de Barcelona de las elecciones municipales de 2015 a través de un proceso de primarias abiertas en el que cualquier ciudadano de Barcelona puede manifestar su opinión votando (2). 
Este interés del PSC-PSOE por la participación ciudadana queda ya patente en un acto de precampaña en las elecciones al Parlament de Catalunya de 2010. En esa época el partido era consciente que existía una sociedad catalana en la que siete de cada diez personas utilizaban Internet, gracias a la Encuesta Territorial de Equipamiento en el Hogar (3). José Montilla, candidato del PSC-PSOE a la presidencia de la Generalitat de Catalunya en ese momento, teniendo en cuenta esos datos, dejó clara su posición en cuanto a la relación que quería mantener con los grassroots: "El mensaje no vendrá dado desde la dirección socialista porque no quiero soldados digitales, sino "activistas críticos y creativos" (4).

Más tarde, tras las elecciones y según un estudio post electoral del $C / S$, se demostró que el 19\% de la población de Barcelona (1.047.117 de personas) siguió la campaña a través de Internet; de este 19\%, el 36,2\% (335.077 personas) lo hizo a través de Facebook, Twitter, etc. Y eso sólo en la provincia de Barcelona (5).

A menos de un mes del inicio de la campaña electoral de las elecciones al Parlament de Catalunya de 2010, el 18 de octubre de ese año, se realizó la primera entrevista a políticos en Twitter. A este acto se le denominó Twittervista (6) y estaba organizado por la asociación STIC.CAT (7). A esta entrevista fueron invitados los candidatos de todos los partidos del arco parlamentario que se presentaban a esos comicios. Ni el cabeza de cartel del PSC-PSOE, José Montilla, ni el de CiU, Artur Mas, acudieron al acto. En representación de estos partidos asistieron segundas figuras; Marina Geli, por parte del PSC-PSOE y Joana Ortega, por la de CiU. La entrevista tuvo tantas demandas de preguntas que el servidor no dio abasto. La organización no esperaba tanta participación ciudadana.

La ciudadanía siguió manifestando su opinión y en la primavera de 2011, el día 15 de mayo, una semana antes de las elecciones municipales en España, un movimiento cívico de protesta denominado 15M ocupó, en primer lugar, la Plaza del Sol en Madrid y, más tarde, muchas más plazas de España (Ginesta, 2013b), como también la Plaza de Cataluña, en Barcelona. Una de las particularidades de este movimiento cívico de protesta es que utilizó las redes sociales tanto para organizarse y comunicarse entre sí, como para hacerlo con el resto de la ciudadanía y los medios de comunicación. Según Enric Casas, encargado de comunicación del PSC-PSOE desde 1982, estas protestas influyeron notablemente en las elecciones municipales al Ayuntamiento de Barcelona, caso de este estudio, marcando la estrategia de comunicación 2.0 y la de la campaña convencional del PSC-PSOE en las elecciones al Ayuntamiento de Barcelona (8).

Antonio Gutiérrez-Rubí, entre otros expertos en comunicación 2.0, definió las elecciones al Congreso de los Diputados del 2011 como las primeras "twitelecciones" (9). Muestra de este hecho fue la "twitterentrevista" que Alfredo Pba hacer Twiterentrevista en 2011 para las elecciones generales a. Para Enric Casas encargado de comunicaciérez Rubalcaba concedió el 11 de noviembre de 2011 utilizando la etiqueta \#yorespondo.

Dos años dos después de las elecciones autonómicas de 2010, punto de partida de este artículo, el escenario en Cataluña era excepcional. A las elecciones al Parlament de Catalunya de 2012 no sólo le precedieron las protestas del movimiento $15 \mathrm{M}$, sino también la manifestación del 11 de septiembre de 2012. Ese día, según $L a$ Vanguardia, 1,5 millones de personas salieron a la calle para pedir la independencia de Cataluña (10).En este contexto de malestar social y desconfianza institucional, y habiéndose convocado una huelga general justo en medio del periodo electoral, el PSC-PSOE afrontó una nueva campaña desde la oposición al Parlament de Catalunya de 2012 (11).

\section{OBJETIVOS Y METODOLOGÍA}

El objetivo principal de este estudio es analizar la relación existente entre el PSC-PSOE, los ciberactivistas afines al partido y la sociedad civil a partir de los mensajes publicados en Twitter por el partido y sus líderes en las campañas electorales transcurridas entre 2010 y 2012 en Cataluña: al Parlament de Catalunya de 2010, al Ayuntamiento de Barcelona, al Congreso de los Diputados de Madrid y, finalmente, al Parlament de Catalunya en 2012.

El periodo de observación de esta investigación incluye los 15 días de cada campaña electoral transcurridas entre 2010 y 2012 en Cataluña. Se excluye de la observación el día de reflexión y el propio de las elecciones.

Para realizar este estudio se elije a PSC-PSOE, que es el principal partido de la oposición en la legislatura 
2006/2010 (12), inicio de esta investigación, en el Parlament de Catalunya.

Los perfiles oficiales de PSC-PSOE en Twitter que se analizan en las diferentes campañas electorales entre 2010 y 2012 son: @fetsandtwitts (cuenta del candidato José Montilla) y @socialistes_cat,en las elecciones al Parlament de Catalunya de 2010; @Hereu2011 y @socialistes_cat, en las elecciones al Ayuntamiento de Barcelona; @CarmeChacon2011 y @socialistes_cat, en las del Congreso de los Diputados y, finalmente, @pere_navarro y @socialistes_cat,en la autonómicas de 2012. Representan, por tanto, el perfil del candidato por un lado y el perfil del partido, por el otro.

En esta investigación se utiliza una triangulación metodológica (Bericat, 1998:60) entre el análisis de contenidos de estos mensajes en Twitter, la revisión bibliográfica y las entrevistas en profundidad y semiestructuradas. El análisis de contenidos está dividido en dos partes, una cuantitativa y una cualitativa (Bericat 1998:60).

La metodología aplicada para la extracción de datos dota de un carácter etnográfico (Soler, 2011:194) a este artículo, ya que se estudia el comportamiento de los políticos y los electores en las diferentes campañas acontecidas en Cataluña entre 2010 y 2012. El análisis de datos cuantitativos se basa en una metodología ya utilizada en el estudio "Observatorio Barça 2010. La campaña del FC Barcelona en la prensa deportiva y las redes sociales" (Ginesta, Gómez, Serrat, 2011). Para obtener la información, se observa durante el periodo electoral cuántos tweets (13) hay en cada perfil, a cuántas personas sigue (14) el propietario del perfil y cuántos seguidores (15) posee la cuenta. Desde el punto de vista cualitativo, cada mensaje de los diferentes perfiles oficiales del PSC-PSOE se analiza según la tipología (nuevos, de respuesta o retweets) o quién es el emisor del mensaje (propietario, equipo de campaña, relacionado con el partido o sin relación con él).

Esta investigación cuenta con entrevistas semiestructuradas (Soriano, 2007: 247) a JordinaFreixanet, Enric Casas, Raquel Quero y Aroa Arauzo, todos ellos responsables de la campaña 2.0 de PSC-PSOE en las diferentes elecciones analizadas. El objetivo de estas entrevistas es contrastar las acciones y decisiones tomadas por estos responsables sobre la comunicación política 2.0 del PSC-PSOE y compararlas entre ellas y con lo que sucedió.

En este artículo también se pueden encontrar cuatro entrevistas en profundidad (Soriano, 2007: 249) a Toni Aira, José Antonio Donaire, Guillem López-Bonafont y Joaquim Nadal. Con estas entrevistas, nuestro objetivo es analizar las dinámicas con las que se trabaja desde el PSC-PSOE en este tipo de comunicación en la época que transcurre la investigación. No solo eso, sino comparar esa forma de trabajar con la de otros partidos a nivel internacional y políticos de referencia, como Barack Obama y su Partido Demócrata en los Estados Unidos.

En esta investigación se realiza una observación no participativa (Soriano, 2007; 229) con el objetivo de recoger datos sobre el funcionamiento y la retransmisión de la información emitida desde un mitin en las redes sociales. En esta situación tan particular se puede observar la estructura del equipo de comunicación 2.0 y su funcionamiento. También se puede observar cuántos y cómo están distribuidos los cibercolaboradores, si hay órdenes del partido o plena libertad a lo que comentar y si hay jerarquización entre los componentes de los ciberactivistas.

\section{EVOLUCIÓN DE LA COMUNICACIÓN POLÍTICA: DE LA 1.0 A LA 2.0}

\subsection{La transformación del ciudadano: de lectores a creadores del discurso público}

El sociólogo crítico Zygmunt Bauman (2010) define la sociedad moderna como líquida. Una sociedad donde las condiciones de actuación de sus miembros cambian antes de que las formas puedan consolidarse en unos hábitos y en una rutina determinada. Por tanto, los triunfadores en esta sociedad son las personas ágiles, ligeras y volátiles como el comercio y las finanzas. Personas hedonistas y egoístas que ven la novedad como una buena noticia, la precariedad como un valor, la inestabilidad como un ímpetu y lo híbrido como una riqueza.

De estos cambios en la sociedad también hace referencia Xavier Peytibi (2011: 228) afirmando que ya en 2010 el mundo está en red, la sociedad ha cambiado y los partidos tienen que adaptarse. Nos encontramos en un contexto en el que la comunicación y la creación de contenidos políticos no dependen sólo de los partidos o de 
los medios, sino que cualquier persona puede convertirse en creador. Según Marçal Sintes(2011:174), antes de todos estos cambios y de la aparición de los medios de comunicación de masas, los rostros de los que estaban en el poder eran prácticamente desconocidos. El poder se demuestra y representa de forma restringida y, prácticamente siempre, este poder era capaz de mantener el control sobre su visibilidad. Con los medios de comunicación de masas, afirma el profesor Sintes (2011: 179),y mencionando a Thompson, los políticos están sometidos a una visibilidad compulsiva creada por la conjunción entre los medios de comunicación de masas y los sistemas democráticos. Tras estos periodos, la ciudadanía quiere opinar, conversar y sentirse partícipe de una comunidad; se siente capacitada y es lo que demuestra constantemente en sus relaciones en la red. Se olvida el espíritu y las acciones relacionales que Internet permite y solo se suele difundir información repetida (Peytibi, 2011: 228). No basta con tener presencia en la red (Twitter, Facebook...) mientras lo que se haga allí sea simplemente esto: generar ruido. Si los activistas se sienten escuchados y ven que lo que hacen como activistas anónimos tiene repercusión en la gente que les rodea y en el bien del candidato o del partido, es entonces cuando se sienten cobijados por una comunidad de personas afines. La ciudadanía quiere cada vez menos ruido y menos propaganda. Quiere comunicación directa y soluciones, relaciones personales (online, pero sobre todo en persona), e incluso poder dar sus ideas y que sean escuchadas. $Y$ quiere que la comunicación sea rápida, pero también sencilla, de tú a tú, amable, que responda a sus dudas a través de sus preguntas o proactivamente, pero que no se base en informaciones generales o solo en críticas, al contrario. Queda atrás la teoría del Two-step Flow of Communication de Katz y Lazarsfeld (1964), en la que afirman que los receptores sólo se forman una opinión tras haber escuchado a aquellas personas que ejercen influencia sobre ellas.

\subsection{Los políticos ante la ciberdemocracia}

Tanto Roc Fages (2008) como Carlos Scolari (2006) y Hugo Pardo (2006), hablan del concepto inteligencia colectiva. Toni Aira (2011) lo denomina "ciberdemocracia". Para Aira (2010: 21), la comunicación política vive un proceso acelerado, no tanto de cambio como de acentuación de las tendencias iniciadas en la ya veterana "era de la televisión". Para él, la "ciberdemocracia" aún es más un sueño que no una realidad, pero tampoco hace falta prescindir de ciertas experiencias e indicadores que apuntan a ello, aunque sea de lejos.

Roc Fages (2008), consultor de innovación, estrategia y trabajo en red, en su artículo "Actitud 2.0: la política más allá de los blogs", afirma que el uso de las herramientas 2.0 potencia la capacidad de los políticos de abrirse, de hablar con la ciudadanía y de escucharla compartir sus opiniones. Una de estas herramientas 2.0, Twitter, es analizada por Piscitelli (2011:15) afirmando que es la red social que, actualmente, más relevancia ha adquirido entre la clase política y periodística. Asegura que se ha convertido "en uno de los mecanismos de comunicación más poderosos de la historia" (16). Y, según José Luís Orihuela, profesor de la Universidad de Navarra, "ha cambiado la red y ha completado el giro social que iniciaron los blogs a finales de los años noventa".

Y es que el objetivo central de los partidos políticos actuales, según Josep Maria Vallès (2000: 363), citando a Kirchheimer, es la movilización de los votantes con ocasión de cada consulta electoral. Con este fin, el partido difumina sus programas, reducidos a menudo a afirmaciones de principio (justicia, orden, cambio, progreso, seguridad, etc.) y moduladas de acuerdo a conveniencias de tipo estratégico. Sin abandonarla enteramente, el partido también desdibuja su conexión preferente con un determinado sector social y pretende captar apoyos electorales en todos los sectores de la sociedad: de ahí el sobrenombre de catch-all-parties o partidos "atrápalo todo". Ya no se trata tanto de transmitir una doctrina política a una gran masa de afiliados; es conseguir su voto.

Gabriel Colomé (1989: 216) identifica algunas características de los partido catch-all en el PSC-PSOE por la forma de financiarse. Esto es debido, según Colomé, a la tasa tan baja de afiliación que posee este partido y a la imposibilidad de autofinanciarse debido a ella. El profesor Gabriel Colomé también encuentra rasgos de partido de masas en el PSC-PSOE. Él explica que esto es debido a dos razones. La primera, por la forma de organizarse, ya que su estructuración se basa en diferentes escalones: local, federación y nacional. La segunda razón que argumenta Gabriel Colomé para considerar al PSC-PSOE como partido de masas es la manera de garantizar la democracia interna; a pesar de que su sistema electoral proporcional hace que se atenúe y que prevalezca la organización nacional (central) sobre las otras. 
Según Albert Medrán (2011: 220), por lo común, ni partidos ni instituciones tienen en sus genes esa predisposición al diálogo. Dependen mucho de liderazgos y apuestas personales hacia una mayor transparencia, hacia una mayor apertura; y chocan con una resistencia interna que se niega a aceptar que las instituciones y los partidos deben ser abiertos y transparentes. Pero la opacidad persiste, no sólo en la alimentación de un debate que va cayendo por su propio peso -el de estar presentes o no-, también en rutinas, gestos y actitudes que muestran que, en esencia, la política es muy poco 2.0. El modo de organizar los partidos, la toma de decisiones... incluso la participación en base a consignas y no a ideas, es la muestra de ello.

Fages-Ramió (2008) afirma que en la aplicación de la Política 2.0, desde el punto de vista de los políticos españoles, pocos han entendido esta actitud de conversar con la ciudadanía y muchos consideran su presencia como una forma más de estar en la red.

A diferencia de lo que realizan los partidos de EEUU, pioneros y de referencia en la comunicación política 2.0, como el Partido Demócrata encabezado por Barack Obama. El profesor de l'Emerson College de Boston, y verdadero impulsor del concepto grassroots, Gregory Payne (2008), estudia este caso y afirma que el emisor y el receptor, voluntariamente, participan en el contacto y el intercambio de mensajes. El objetivo de estos encuentros de comunicación deliberativos no es necesariamente llegar a un acuerdo sobre todas las cuestiones, sino empezar la conversación y, aún más, el entendimiento entre las partes en conflicto. Con más confianza entre las partes, se aprende a comenzar a aceptar, en lo posible, las diferencias de perspectivas, como se explora más áreas en las que existe un acuerdo y los valores compartidos.

Enric Ordeix y Xavier Ginesta (2011), en su artículo "Beyond the Votes: A European Perspective on the Use of Public Relations to Legitimize Authority in Obama's Campaign", también hablan del concepto grassroots. Para Ginesta y Ordeix, el Presidente electo Obama fue el primer candidato presidencial que confiaba en la movilización de bases a través de tecnologías de la información y la comunicación (TIC) para crear complicidades con los votantes indecisos y otras partes interesadas. En esta campaña presidencial de 2008, las TIC (por encima de todo, las comunidades virtuales) se convierten definitivamente en una herramienta esencial de marketing político que deben ser tenidas en cuenta por los gerentes de relaciones públicas y los directores de comunicación de campañas futuras.

En un artículo publicado en la web del thinktank Terra Nova (17), el director de campaña de Obama, Olivier Ferrand, se refiere a las claves de esta gran movilización. Una de ellas fue el hecho de ser una campaña horizontal. Los 2.700 asalariados de la campaña no eran los emisores de una comunicación que sólo iba de arriba abajo, sino que su tarea era coordinar los militantes voluntarios y sus iniciativas. Otra, también muy importante, fue que el militantismo (el trabajo del militante) era organizar a la carta según el perfil y las ganas de trabajar de cada uno de los que se habían inscrito (Costa, 2009:36).

\section{RESULTADOS Y TRABAJO DE CAMPO}

La presencia del PSC-PSOE en Twitter en las campañas electorales en Cataluña comprendidas entre 2010 y 2012, no varió. En todas ellas el partido contó con dos cuentas oficiales; la del candidato y la propia del partido. Algo que sí que varió fue la forma de denominar las cuentas.

En las elecciones al Parlament de Catalunya de 2010, la cuenta del candidato se denominaba Fets and twitts y el nombre de usuario era @fetsandtwitts. Ni en el título de la cuenta, ni en el nombre del usuario (18) se hacía referencia al candidato, solo el avatar (19) contenía una foto de José Montilla. El perfil del partido se encabezaba con Socialistas (PSC) y su nombre de usuario era @socialistes_cat. El avatar de esta cuenta era el logotipo del PSC.

En las elecciones al Ayuntamiento de Barcelona de 2011, el perfil del candidato se denominaba Jordi Hereu 2011 y el nombre de usuario era @Hereu2011. La cuenta iba acompañada por una foto del candidato (avatar). En el caso del partido, se repetía exactamente el nombre y avatar de las elecciones del 2010.

Para las elecciones al Congreso de los Diputados de 2011, la cuenta del candidato para el PSC-PSOE se denominaba Carme Chacon 2011 y el nombre de usuario era @CarmeChacon2011. En este perfil también se podía ver en el avatar una foto de la candidata. Para estas elecciones, el perfil del partido siguió con la misma 
estrategia que en las dos anteriores.

Finalmente, en las elecciones al Parlament de Catalunya de 2012, el PSC-PSOE presentaba el perfil del candidato con una foto de él y con el título Pere Navarro Morera. El nombre de usuario para esta ocasión era @pere_navarro. En el perfil público del partido se cambió la estrategia por primera vez. El título y el nombre de usuario se mantuvieron, Socialistes (PSC) y @socialiestes_cat. En cambio, el avatar varió. Se pasó del logotipo del PSC al texto: L'Alternativa sensata.

En las elecciones al Parlament de Catalunya de 2010 se emitieron 937 mensajes desde los perfiles oficiales del PSC-PSOE en Twitter; 247 desde el perfil del candidato y 690 desde el perfil del partido. De estos 937, 725 fueron mensajes nuevos (242 de @fetsandtwitts/ 483 de @socialistes_cat), 69 son retweets (0 de @fetsandtwitts/ 69 de @socialistes_cat)y 143 fueron mensajes de respuesta (1 de @fetsandtwitts/ 142 de @socialistes_cat).

En los siguientes comicios, en los del Ayuntamiento de Barcelona de 2011, se emitieron desde los perfiles del PSC-PSOE en Twitter 582 mensajes; 416 desde la cuenta de Jordi Hereu y 166 desde la del partido. De estos 582 tweets,455 fueron mensajes nuevos (350 @Hereu2011/105 @socialistes_cat), 53 de respuesta (51 @Hereu2011/ 2 @socialistes_cat) y 74 retweets (68 @Hereu2011/ 6 @socialistes_cat).

El número de mensajes que se emitieron desde los perfiles del PSC-PSOE y de Carme Chacón en las elecciones al Congreso de los Diputados de 2011 fueron de 1.489, 787 desde @CarmeChacon2011 y 702 desde @socialistes_cat. En esta ocasión, de los 1.489 tweets, 733 fueron mensajes nuevos (486 @CarmeChacon2011/ 247 @socialistes_cat), 143 fueron mensajes de respuesta (109 @CarmeChacon2011/ 34 @socialistes_cat) mientras que 613 fueronretweets(190@CarmeChacon2011/ 423 @socialistes_cat).

En las elecciones al Parlament de Catalunya se emitieron desde las cuentas públicas y oficiales del PSCPSOE, 1.693 mensajes; 467 desde@pere_navarro y 1.226 desde @socialistes_cat. De estos 1.693, 978 fueron nuevos mensajes (475 @pere_navarro/ 503 @socialistes_cat), 28 de respuesta (0 @pere_navarro/ 28 @socialistes_cat) y 687 retweets (0 @pere_navarro/ 687 @socialistes_cat).

En las elecciones autonómicas de 2010, el PSC-PSOE contaba con una página web denominada "la blogosfera socialista". En ella se encontraba un resumen de los blogs de los políticos socialistas activos en la red. La página www.sociates.cat, también fue un punto de referencia en el que los ciudadanos se podían informar de lo que estaba pasando en la campaña, así como del programa electoral. En ella se recogían noticias de los actos, vídeos de Youtube, etc.

En 2011, en las elecciones municipales de Barcelona, www.sociates.cat funcionaba de forma muy parecida. En esta página web, los ciudadanos encontraron una campaña denominada "15 dies 15 accions", donde se recomendaba de forma clara durante los quince días de campaña electoral, qué y cómo podían participar los ciberactivistas en la difusión del mensaje político. Por ejemplo, el primer día de campaña, en el perfil del PSCPSOE, se publicaba un mensaje con el siguiente texto:"Comenzamos! Acción 1. Lemas para una campaña”. A esta frase le acompañaba un link que conducía hacia la página web www.sociates.cat.

En las elecciones al Congreso de los Diputados de 2011 no se registró ninguna novedad. En esta ocasión, www.sociates.cat funcionaba de la misma forma que en las elecciones de 2010, un recopilatorio de vídeos, noticias, lemas, etc.

En las elecciones al Parlament de Catalunya de 2012 se detectó una zona específica en www.sociates.cat denominada Ciberactivismo. En ella se animaba a los activistas con el siguiente texto: "Haz campaña. Descarga el material". Cuando se hacía clic en este link, se encontraba material de campaña como los carteles electorales, infografías explicando conceptos del programa electoral, fotos, links a vídeos, etc.

A pie de calle, en los mítines de la campaña electoral del Parlament de Catalunya de 2010, se observó una evolución en la que el PSC-PSOE denominaba zona 2.0. En el momento en que el candidato explicaba su programa, un grupo de 30 personas retransmitía en Internet lo que allí estaba sucediendo en esta zona. Estos ciberactivistas se componían de voluntarios afines al partido y, en alguna ocasión, eran personas referentes en 
el mundo de la comunicación política 2.0, como Saül Gordillo. Este grupo lo dirigía Raquel Quero, persona que ayuda a Jordina Freixenet en las funciones de responsable de comunicación 2.0 en esas elecciones. A su lado se situaba un representante de la federación del lugar en el que se realizaba el acto. Él era el encargado de movilizar a los ciberactivistas de la zona.

En las elecciones al Ayuntamiento de Barcelona y al Congreso de los Diputados de 2011,esta zona 2.0 menguó. Se mantuvo la estructura de una persona que dirigía a un grupo, pero mucho más reducido. Se potenció que los miembros del PSC-PSOE participaran del discurso público en Internet.

En 2012, en las elecciones al Parlament de Catalunya, la zona 2.0 quedó reducida a 4 personas. Sólo en el mitin central de campaña aumentó la cifra. Se invitó al público a comentar desde sus teléfonos móviles lo que estaba sucediendo en los actos.

En todas las elecciones existía un grupo de 20 jóvenes que se reunían durante los 15 días de campaña en la sede del PSC-PSOE para ayudar a difundir el programa y hacerse eco de lo que estaba pasando en Internet por parte del resto de la ciudadanía.

\section{DISCUSIÓN}

El hecho de que @fetsandtweets, la cuenta del candidato del PSC-PSOE, José Montilla, en las elecciones al Parlament de Catalunya de 2010, no hiciera referencia al nombre del candidato puede dar a entender que existía una voluntad de no comunicar en este medio. Tal y como afirma Watzalawick (1981/1967:50): "Es imposible no comunicar. Hasta cuando una persona está en un banco callado mirando al fondo, está comunicado que no quiere comunicar".

Esta actitud y la creación expresa de las cuentas oficiales y la no participación de los candidatos en ellas, sitúa al PSC-PSOE en una posición muy cercana a la de Marçal Sintes (2011:179) cuando afirma, mencionando a Sartori, que los líderes políticos están obligados a convertirse en actores para actuar como él o como sus asesores consideren que se espera que actúe. A esta obligación, Sintes la denomina visibilidad compulsiva. Esta idea queda reforzada con el reconocimiento, por parte de los responsables del equipo de campaña 2.0 de PSC-PSOE durante las elecciones de 2010 a 2012 en Cataluña (Jordina Freixanet, Enric Casas, Raquel Quero y Aroa Arauzo), de que los candidatos participaron poco del discurso público que se da en esta herramienta.

Los candidatos no son los únicos que no participaron demasiado en el discurso público en las redes. En dos años, el número de mensajes emitidos desde las cuentas oficiales del PSC-PSOE se duplicó, mientras que las menciones a ellos por parte de la ciudadanía se multiplicaron por nueve y medio. Dato que confirma la teoría de Peybeti (2011: 228), en la que afirma que cualquier miembro de la sociedad puede ser creador de contenidos políticos. Los 301.948 mensajes de diferencia que emitió la ciudadanía entre las elecciones al Parlament de Catalunya de 2010 y las de 2012, denota la voluntad de ésta de opinar, conversar y sentirse partícipe de una comunidad, algo a lo que hace referencia Marçal Sintes (2011:179). Según Peybeti, la ciudadanía quiere una comunicación directa y ser escuchada, cosa que no se cumple en ese periodo. Los mensajes de respuesta y los retweets, marcadores de interacción del partido con la ciudadanía, que se emitieron desde las cuentas del PSCPSOE en Twitter de 2010 a 2012 se multiplicaron por 3,3; muy lejos del aumento de participación comentado con anterioridad de un 9,5 .

Durante el trabajo de campo se observa que en todas las elecciones en Cataluña, de 2010 a 2012, el PSCPSOE mantuvo al responsable de la federación de la zona a la que acudía el candidato para que dinamizara el discurso político que se emitía desde las cuentas de Twitter y animara a los ciberactivistas de la zona. Forma de organizarse que sitúa al PSC-PSOE, según Gabriel Colomé (1989: 216), como partido de masas a pesar de su forma de financiarse, lo que le convertiría en partido catch-all según el autor.

En el análisis realizado se detecta, por parte del PSC-PSOE, un envío de mensajes con recomendaciones, links que conducen a lemas para ser compartidos y campañas que animaban a difundir mensajes. Esta coordinación de voluntarios tiene mucho que ver con la voluntad de movilización de los afiliados a un partido para conseguir sus votos que utiliza Vallès (2000: 363), citando a Kirchheimer, para definir a los partidos catch-all-parties, o partidos "atrápalo todo". Rasgos que también detecta Colomé, como se ha comentado con anterioridad. 
En relación a todo esto, Enric Casas, responsable de comunicación en las elecciones al Ayuntamiento de Barcelona de 2011, afirma que el PSC-PSOE tiene una concepción de lo que es la política y de lo que hace colaborar a la gente en ella: para él una campaña de éxito, sobre todo si se quiere motivar, es una campaña que funciona por nódulos y redes, no por una ideología clara. Para eso se ha de dar un contenido básico en la red y, después, potenciar la creatividad de la gente. Joaquim Nadal, ex diputado del PSC-PSOE, coincide con Enric Casas al afirmar que, de no ser así, la gente se da cuenta que lo que se busca es una consigna simple. "Si la repites como un lorito, toda la fuerza de esa gente, de la familia política, pierde el sentido". En cambio, asegura Nadal, "si tu apuntas una idea sobre alguna cosa que te parece bien, tu opinión se hará respetar dentro de Twitter" (20). José Antonio Donaire, ex diputado del PSC-PSOE y experto en comunicación 2.0, coincide con ellos pero cree que los partidos políticos están actuando como creadores de contenidos y hacen lo posible para trasmitir los mensajes unidireccionalmente a los ciudadanos (21).

En 2010, según Jordina Freixanet, responsable del equipo de campaña 2.0 en las elecciones al Parlament de Catalunya del mismo año, afirma que los cibervoluntarios (Payne, 2008; Costa, 2009; Ordeix y Ginesta, 2011) podían participar de dos maneras: siguiendo al candidato en los mítines o retransmitiendo el mensaje desde la sede del partido (22). En los mítines de estas elecciones existía una zona 2.0 en la que estos ciberactivistas y gente de referencia en el mundo de la comunicación, como el periodista Saül Gordillo, retransmitían lo que allí está pasando. Esta forma de actuar tiene un referente teórico básico, sobretodo basado en la teoría conocida como Two-step Flow of Communication de Katz y Lazarsfeld (1964), en la que afirman que los receptores sólo se forman una opinión tras haber escuchado a aquellas personas que ejercen influencia sobre ellas. Hasta la zona 2.0 también se acercaron diferentes miembros del partido, como el ex ministro socialista Celestino Corbacho, para mostrar apoyo al candidato.

Durante las elecciones municipales de 2011 y en las del Congreso de los Diputados, la zona 2.0 se redujo en los mítines y casi llegó a desaparecer. En las elecciones al Parlament de Catalunya de 2012, la zona 2.0 no tenía tanto sentido ya que, según Aroa Arauzo, responsable de la campaña 2.0 del PSC-PSOE en 2012: "hasta ahora la gente llevaba sus portátiles, los enchufaban a las mesas y utilizaban la conexión”. Hoy en día, afirma Arauzo, la gente utiliza directamente el móvil y no necesitan estar in situ (23). Estos cambios son un ejemplo de a lo que se refiere el sociólogo Zygmunt Bauman (2010) cuando define la sociedad moderna como líquida: una sociedad donde las condiciones de actuación de sus miembros cambian antes de que las formas puedan consolidarse en unos hábitos y en unas rutinas determinadas.

De los ciberactivistas que acompañaban al presidente en los mítines de la campaña electoral de las elecciones autonómicas de 2010, según Freixanet, surgió la propuesta de resumir su ánimo en una canción para compartirla en Spotify y en Twitter. Más tarde se convirtió en una acción de campaña oficial en las elecciones municipales de 2011. Como se ha mencionado, existieron compañeros que apoyaron esta acción y difundieron el mensaje del PSC-PSOE al máximo. En las elecciones autonómicas de 2012, esta situación cambió ya que Aroa Arauzo reconoce una dificultad en la predisposición a participar por parte de los propios ciberactivistas en esta campaña. Arauzo afirma que la gente estaba desmovilizada en general, tanto a nivel de militantes como de sociedad civil. La responsable de la campaña 2.0 explica que, debido al contexto econa no tuviera ganas de de $\mathrm{n}$ esspredisposiciucho menos con los partidos. na por ms mMiquel Iceta entre otors ciberactivistas se ómico y social, la ciudadanía no tenía ganas de participar, y mucho menos con los partidos. Un ejemplo de esto es que en el perfil @socialstes.cat del PSC-PSOE, el primer día de las elecciones al Parlament de Catalunya de 2012, se retuiteó un mensaje de @pscbarcelona en el que se podía leer: "15 días para cambiar. Buscamos voluntarios".

Todos los candidatos hicieron un reconocimiento y agradecieron la colaboración de los ciberactivistas. Montilla, por ejemplo, en un mensaje desde @fetsantweets les dijo: “Gracias a los que estáis aquí y a los que nos seguís en Internet y en las redes sociales. He aprendido de vosotros". En la cuenta de Jordi Hereu, en 2011, se celebró la llegada a los 2.000 seguidores y se agradeció a todos aquellos que le seguían. En el de Carme Chacón, se agradeció a la red socialista de la ciudad de Badalona por difundir \#noeslomismo, uno de los lemas de la campaña de 2011. Finalmente, Pere Navarro dio las gracias a los miles de ciudadanos que habían contribuido a difundir la \#alternativasensata. Éste fue uno de los pocos mensajes firmados con PNM, señal que lo había escrito el mismo Pere Navarro. 
La ciberdemocracia (24), la actitud de la ciudadanía en la red, no siempre es buena. El aumento de la participación ciudadana hace también que aumenten actitudes incorrectas. Un ejemplo de esto es el que reconoce Aroa Arauzo durante las elecciones al Parlament de Catalunya de 2012 y que consistió en amenazas de muerte realizadas a Pere Navarro y a su familia. No se registra ningún ejemplo de éstas ya que, según Arauzo, todos estos mensajes fueron eliminados de la cuenta. Un claro ejemplo de esta mala praxis es el de Elena Valenciano, número dos del PSOE en 2013. El 2 de marzo de ese año, Valenciano publicó en su cuenta de Twitter "Dentro de media hora saldré de Twitter. Algun@s han empezado a perseguir a mis hij@s, y ese sí es el límite". "Twitter ha cambiado mucho en estos años... Contra mí, era normal. Contra mis hij@ es inmoral". "Desde aquí no hay manera de defenderles. Me voy para poder protegerles. Adiós" (25).

\section{CONCLUSIONES}

En el PSC-PSOE ha habido una evolución en relación con la manera de implicar a los grassroots e incrementar la participación ciudadana. El número de retweets y de mensajes de respuestas aumentó, de 2010 a 2012, multiplicándose por 3,5. Esta evolución no es suficiente ya que la ciudadanía demostró una mayor voluntad de participación y relación con los políticos, aumentando en 9,5 los mensajes en referencia al partido y sus candidatos.

La libertad en la construcción del discurso político de los ciberactivistas, pretendida por los responsables de campaña 2.0 de todas las elecciones, se hizo patente en la manifestación de José Montilla en un acto de precampaña de las elecciones al Parlament de Catalunya de 2010 con la siguiente afirmación: "El mensaje no vendrá dado desde la dirección socialista, porque no quiere soldados digitales, sino activistas críticos y creativos" (26). Esta voluntad hizo que el mensaje que se emitió desde las cuentas de Twitter quedara difuminado y poco concreto. Da la sensación, como afirma Jordina Freixanet, responsable de la campaña del partido en las elecciones al Parlament de Catalunya de 2010, de "ejército de Pancho Villa".

EI PSC-PSOE evolucionó con los grassroots recogiendo ideas de sus ciberactivistas y transformándolas en acciones de campaña, dándoles así más protagonismo. No solo eso, sino que intentó concienciar a los miembros de sus listas de lo importante de su presencia en la red, como reconoce Aroa Arauzo, responsable de la campaña de las elecciones al Parlament de Catalunya de 2012.

Por último, y debido a la coyuntura social y por los cambios tecnológicos, los espacios destinados a la retransmisión de mítines tienden a desaparecer. También la presencia de los ciberactivistas en la sede del partido. Ahora, cada vez más, con la aparición de los teléfonos móviles, los asistentes como público a los actos de campaña se convierten en los creadores de mensajes.

\section{BIBLIOGRAFÍA}

Aira, T. (2010): La comunicació política.Barcelona:UOC.

Aira, T. (2011): Los nuevos profesionales de la "democracia mediática". Perfiles y roles emergentes en los equipos de comunicación y estrategia políticos., X Congreso: Política en Red del 7 al 9 de septiembre de 2011. Murcia: Asociación Española de Ciencia Política y de la Administración (AECPA) [online] http://www.aecpa.es/congresos/10/ponencias/14/ [Fecha de consulta: 12 de diciembre de 2013]

Bauman, Z.\& Santos, A.(2010): Vida líquida, Estado y Sociedad. Barcelona: Paidós.

Berger, P., \& Luckmann, T. (1968):La construcción social de la realidad . Buenos Aires:Arromortu.

Canel, M. (1999):Comunicación política. Técnicas y estrategias para los cociedad de la información. Madrid:Tecnos.

Colomé, G. (1989): El partit dels Socialistes de Catalunya: estructura, funcionament i electorat (1978-1984). Barcelona: Edicions 62.

Costa, P.O. (2009) "La utilització d'internet per part de Barack Obama transforma la comunicació política" Quaderns del CAC. Vol.33 (Diciembre 2009), pp.35-41. 
De Moragas, M. (2011):Interpretar la comunicación .Barcelona: Gedisa.

Fages-Ramió, R. (2008) "Actitud 2.0: la política más allá de los blogs". IDP. Revista de Internet, Derecho y Política,[En línea], núm. 7, (septiembre 2008), pp. 22 http://www.uoc.edu/idp/7/dt/esp/fages.pdf (Fecha de consulta 25 de junio 2012).

Franco, D. "El análisis de la participación digital de los grassroots en las campañas electorales de CiU en Cataluña”, En III Jornades Internacionals de comunicació i societat del 8 de noviembre de 2013. Girona: Universitat de Girona.

Freire, J. Gutiérrez-Rubí, A. (2010):32 Tendencias de cambio" LABO. Barcelona: Laboratorio de Tendencias [En línea]. http://www.gutierrez-rubi.es/32-tendencias-de-cambio-2010-2020/ (Fecha de consulta: 25 de junio 2012)

Ginesta, X.(2013a.): "La Catalunya del plebiscit. El futur està en joc: de l'Onze de Setembre al 25 de novembre, Curber Edidions, Girona.

Ginesta, X. (2013b): "Xarxes socials i nova política”. Acta de la conferencia E-week del 5 de noviembre de 2103. Centelles: E-week.

Ginesta, X. \&Ordeix, E.: "Beyond the Votes: A European Perspective on the Use of Public Relations to Legitimize Authority in Obama's Campaign". American Behavioral Scientist. Vol, 55 (Abril de 2011) [online] http://abs.sagepub.com/content/55/6/683. [Fecha de consulta: 12 de febrero de 2014]

Ginesta, X., Gómez, M. \& Serrat, J. (2011):“Observatorio Barça 2010. La campaña del FC Barcelona en la prensa deportiva y las redes sociales”, Universitat de Vic.

Giral, E. (1989):Partit dels Socialistes de Catalunya, PSC-POSE. Cronologia (1978-1999). Barcelona: ICPS.

Gordillo, S.: “Comunicació i política: el pas de l'1.0 al 2.0”. Trípodos, vol. 27, (marzo de 2011) P.175-188.

Katz, E., \& Lazarsfeld, P.(1964):Personal Influence; The part played by people in mass commnunications.Illinois: G. F. Press.

Laswell, H. (1948): The structure and Fuction of comunication in society. New York, Harper.

McCombs, M. \& Shaw, D.(1997): The Emergence of American Political Issues: The Agenda Setting Function of the Press.West. Pub. Co, T. St.Paul.

McQuail, D. (2000):Introducción a la teoría de la comunicación de masas, 5th Edition, Barcelona:Paidós.

Medrán, A.: No preguntes. No digas. En Cotino L. (editor), (2010): Libertades de expresión e información en Internet y las redes sociales: ejercicio, amenazas y garantías, [online] Valencia, PUV (Publicaciones de la Universidad de Valencia),pp. 220-222.

Orihuela, J. (2010):Mundo Twitter. Barcelona:Alienta.

Peybiti, X.: Las relaciones políticas online y el ruido en campaña. En Cotino, L. (2010): Libertades de expresión e información en Internet y las redes sociales: ejercicio, amenazas y garantías, [online] Valencia, PUV (Publicaciones de la Universidad de Valencia),pp. 226-231.

Piscitelli, A. (2011). Prólogo: Twitter, la revolución y los enfoques ni-ni. En Orihuela, J.L., Mundo Twitter (pp. 1520). Barcelona: Alienta.

Scolari. C., 2006, "Web 2.0 Caos conceptual y nuevos mitos en el discurso cibercultural. IX Congreso Grupo de Trabajo: Cibercultura y nuevas tecnologías de la información". IX Congreso IBERCOM EI espacio iberoamericano de comunicación en la era digital, En línea, http://scholar.google.es/scholar? start=10\&q=pol\%C3\%ADtica+2.0\&hl=es\&as_sdt=0\&as_vis= fecha de consulta [Fecha de consulta: 12 de febrero de 2014] 
Sintes, M. (2011): Periodistes contra politics. Barcelona: Columna Edicions.

Soriano, J. (2007):L’ofici del comunicòleg. Mètodes per investigar la comunicació. Vic:Eumo Editorial.

Vallés, J. (2000):Ciencia Política. Una introducción, 8th. Barcelona: Edition, Ariel.

Watzalawick, P.(1967/1981): Teoría de la comunicación humana: interacciones, patologías y paradojas. Barcelona: Herder.

Xifra, J.: "Los thinktank y advocacytank como actores de la comunicación política ", Anàlisi, vol. 32, (2005) pp.73-91 http://ddd.uab.es/pub/analisi/02112175n32p73.pdf. [Fecha de consulta: 12 de enero de 2014]

\section{Breve semblanza del autor}

Daniel Franco es profesor de comunicación 2.0 en la Universidad de Turismo Sant Ignasi (Universitat Ramon Llull / ESADE) y en la Universitat Internacional de Catalunya. Ha trabajado como reportero gráfico durante 10 años en los informativos de TV3. En 2010 realiza el máster de Comunicación Digital e Interactiva de la Universidad de Vic y actualmente se está doctorando en la misma.

(1) Baquero, A; Márquez, C. "Cataluña clama por la independencia" El Periódico [online], 11 de septiembre de 2012, Barcelona, http://bit.ly/1xtiDNF, [Fecha de consulta: 12 de septiembre de 2012]

(2) Rico, J. "EI PSC se encomienda a Collboni para recuperar la alcaldía de Barcelona" El Periódico [online], 6 de abril de 2014, Barcelona, http://bit.ly/1k2uriP, [Fecha de consulta: 21 de abril de 2014]

(3) Idescat. Hogares con acceso a Internet, 2010. Instituto de estadística de Cataluña, 23 de diciembre de 2010. http://bit.ly/1owYYdW [Fecha de consulta: 04 de enero de 2011]

(4) Maiol, R. "Montilla llama a movilizarse a sus militantes en Internet”. El País [online], 22 de mayo de 2010. http://bit.ly/OeBtVE [Fecha de consulta: 03 de febrero de 2014]

(5) Centro de Investigaciones Sociológicas. Estudio post electoral de las elecciones autonómicas al Parlamento de Cataluña de 2010. Centro de Investigaciones Sociológicas , 03 de diciembre de 2010. http://bit.ly/1ly3djr [Fecha de consulta, 13 de febrero de 21012]

(6) EFE [online]. "Los candidatos de los partidos catalanes participarán en un debate en Twitter", La Vanguardia, 13 de octubre de 2010, [online] http://PSC-PSOE.lavanguardia.com/elecciones-catalanas2010/20101013/54022825774/los-candidatos-de-los-partidos-catalanes-participaran-en-un-debate-entwitter.html [Fecha de consulta: 14/10/2010).

(7) La asociación STIC.CAT (Societat de Tecnologia i Coneixement ) nace en el año 2008 con la vocación de ser una entidad que genere la interactividad y la innovación en el contexto de la cultura y la lengua catalana con la utilización de las tecnologías de la información y la comunicación como eje vertebrador.

http://www.stic.cat/que-es-stic/

(8) Entrevista a Enric Casas realizada el 24 de diciembre de 2014 en Barcelona.

(9) Gutiérrez-Rubí, A. "La batalla de Twitter" El País [online], 8 de noviembre de 2011, http://bit.ly/QHBsf8 [Fecha de consulta: 4 de febrero de 2014]

(10) Política. "Masiva manifestación por la independencia de Catalunya", La Vanguardia, 11 de septiembre de 2011, [online], http://bit.ly/1osUxjU

(11) El País, "Cientos de miles de manifestantes cierran la protesta de la huelga general del 14-N" El País 
[online], 14 de noviembre de 2012, Madrid, http://bit.ly/1osUtkb [Fecha de consulta: 4 de febrero de 2014]

(12) La Vanguardia [online], Histórico de resultados electorales en Catalunya (1977-2010), La Vanguardia, 11 de noviembre de 2010, [online] http://bit.ly/PnBdVs

(13) Es un mensaje ó actualización de Twitter.

(14) Es cuando te suscribes a las actualizaciones de algún usuario dentro de Twitter.

(15) Son aquellos usuarios que están suscritos a tus actualizaciones o tweets.

(17) Ferrand, O; Montebourg, A. (2009) "Fromcampaigntogovernance". Març París: Terranova, 2007. [En línea] citado por Costa, P.-O. "La utilització d'internet per part de Barack Obama transforma la comunicació política" Quaderns del CAC 33, 2009:36

(18) La diferencia es el título o nombre de la cuenta y el nombre de usuario va acompañado de @. Puede ser diferente.

(19) Avatar: Es la imagen de cada usuario. Acompaña a cada uno de nuestros tweets y nos representa en Twitter se trata de una imagen cuadrada de pequeño tamaño que no puede ser ofensiva en manera alguna ni violar ningún tipo de derecho de autor.

(20) Entrevista a Joaquim Nadal realizada el 16 de octubre de 2012 en el Parlament de Catalunya.

(21) Entrevista a José Antonio Donaire realizada en la Universitat de Turisme de Girona el día 22 de octubre de 2012.

(22) Entrevista a JordinaFreixanet realizada el 13 de febrero de 2011 en la sede del PSC-PSOE en Barcelona.

(23) Entrevista a Aroa Arauzo realizada el 19 de febrero de 2013 en la sede del PSC-PSOE en Barcelona.

(24) Carlos Scolari (2006), Hugo Pardo (2006) y Toni Aira (2010:21)

(25) Sánchez, M. "Elena Valenciano deja el Twitter por los ataques a sus hijos: "La indefensión es total", El Mundo [online], 2 de marzo de 2013. http://mun.do/RHJAN8 [Fecha de consulta: 05 de marzo de 2013].

(26)Maiol, R. "Montilla llama a movilizarse a sus militantes en Internet". El País [online], 22 de mayo de 2010. http://bit.ly/OeBtVE [Fecha de consulta: 03 de febrero de 2014].

Ámbitos. Revista Internacional de Comunicación, n.26, año 2014, tercer trimestre (otoño).

Recibido: 25/6/2014

Aprobado: 28/7/2014 